\title{
Primary metabolic processes as drivers of leaf ageing
}

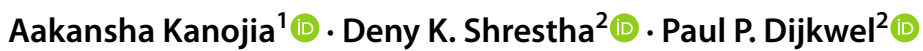

Received: 14 March 2021 / Revised: 29 June 2021 / Accepted: 29 June 2021 / Published online: 19 July 2021

(c) The Author(s) 2021

\begin{abstract}
Ageing in plants is a highly coordinated and complex process that starts with the birth of the plant or plant organ and ends with its death. A vivid manifestation of the final stage of leaf ageing is exemplified by the autumn colours of deciduous trees. Over the past decades, technological advances have allowed plant ageing to be studied on a systems biology level, by means of multi-omics approaches. Here, we review some of these studies and argue that these provide strong support for basic metabolic processes as drivers for ageing. In particular, core cellular processes that control the metabolism of chlorophyll, amino acids, sugars, DNA and reactive oxygen species correlate with leaf ageing. However, while multi-omics studies excel at identifying correlative processes and pathways, molecular genetic approaches can provide proof that such processes and pathways control ageing, by means of knock-out and ectopic expression of predicted regulatory genes. Therefore, we also review historic and current molecular evidence to directly test the hypotheses unveiled by the systems biology approaches. We found that the molecular genetic approaches, by and large, confirm the multi-omics-derived hypotheses with notable exceptions, where there is scant evidence that chlorophyll and DNA metabolism are important drivers of leaf ageing. We present a model that summarises the core cellular processes that drive leaf ageing and propose that developmental processes are tightly linked to primary metabolism to inevitably lead to ageing and death.
\end{abstract}

Keywords System biology $\cdot$ Ageing $\cdot$ Primary metabolism $\cdot$ DNA repair $\cdot$ Reactive oxygen species $\cdot$ Molecular genetics

\section{Introduction}

The plant kingdom includes some of the longest living species on earth, with bristlecone pine at a demonstrated lifespan of several thousand years and a clonally reproducing seagrass estimated at a minimum of 80,000 years [1,2]. However, much of the living tissue of each plant ages over a much shorter time, often in response to seasonally driven cold or dry periods. In deciduous trees, this is most spectacularly seen in autumn, when leaves change colour and the subsequently rapid fall of leaf foliage. In fact, it has been

Paul P. Dijkwel

p.dijkwel@massey.ac.nz

Aakansha Kanojia

kanojia@cpsbb.eu

Deny K. Shrestha

denykumarshrestha@gmail.com

1 Center of Plant Systems Biology and Biotechnology, Ruski 139 Blvd., Plovdiv 4000, Bulgaria

2 School of Fundamental Sciences, Massey University, Private Bag 11222, Palmerston North, New Zealand proposed that the short life cycle of leaves and other plant tissue is at the basis of the extreme longevity of the whole organism [3, 4]. Nevertheless, since it is difficult to study whole plant ageing in trees, which live many times longer than the researchers studying the tree, plant leaves are often chosen to study ageing. Here, we review literature on leaf ageing and ageing of short-lived annual plants. The conclusions drawn from such studies, therefore, do not necessarily apply to long-lived plants.

We consider that leaf ageing encompasses the whole lifespan of a leaf, i.e., starting from initiation and ending with the process called leaf senescence. Leaf senescence is the most studied aspect of leaf ageing and involves a highly regulated and complex process requiring a vast set of genes that interact through multiple signalling pathways $[5,6]$. Senescence of individual leaves can take place throughout plant development, but at the end of monocarpic plants' lifes, all leaves senesce at the same time [7]. Senescence of a leaf begins when the photosynthetic rate drops below a certain threshold; therefore, the leaf no longer contributes to carbon fixation [8]. De-greening of the leaves, due to chlorophyll degradation, is a visible indicator of leaf senescence 
[9]. Since, chlorophyll, protein, and membrane degradation occur during senescence, the levels of these parameters are used as markers in the study of senescence progression [10, 11]. The phenomenon of leaf senescence also includes other cellular catabolic processes such as lipid peroxidation and degradation of proteins, carbohydrates, RNA, and DNA $[8,12]$. The dismantling of cellular components during the senescence process releases a considerable amount of nutrients, which are then remobilised to the growing parts of the plant $[13,14]$. Thus, leaf senescence is an active and destructive process but essential for development of actively growing organs such as new leaves, seeds, and buds that need to survive through winter $[8,15-17]$.

Recent technological and computational 'omics' advances have allowed researchers to take a more holistic approach to studying the regulation of senescence processes. However, the events leading to the senescence process are poorly understood. Here, we review computational studies and analyse outcomes relevant to leaf ageing, to refine hypotheses that describe processes that may drive leaf ageing. While computational studies excel at sifting through large datasets to identify potential regulatory hubs and processes, hypotheses developed through these studies often need to be tested using genetic methods that perturb those processes, like gene knock-out or overexpression studies. Thus, we subsequently examine evidence that supports or rejects computer-based hypotheses. Finally, we summarise and highlight general mechanisms of leaf ageing, thereby largely overlooking ageing processes that are species-specific or that affect the onset of senescence due to reproduction or a changing season in monocarpic and deciduous plants, respectively. Nevertheless, since ageing is such a universal process, we expect that general mechanisms are conserved throughout the plant kingdom.

\section{Systems biology approaches of ageing suggest primary metabolic processes as major age-regulating factors}

Various 'omics' studies in the field of plant ageing have helped develop hypotheses to explain the complex plant ageing system $[19,20]$. Nevertheless, these studies mainly illustrate changes in biological pathways during natural or stress-induced senescence $[18,20]$. Genomics research has contributed to understanding the genetic composition, function, and potential mechanism of the genes that regulate ageing and senescence in plants [20]. Transcriptomics has provided extensive information about the gene expression patterns in ageing tissue and while it cannot accurately predict the metabolome, it provides background information for metabolomics studies [21-23]. Metabolomics, while generally less comprehensive, may potentially be more informative than genome and gene expression studies because metabolites constitute the end-product of all cellular functions, and thus take into account complex enzymatic pathways where enzyme activity is modulated by regulatory proteins and the metabolome itself.

First, we review multi-omics studies of ageing leaves, which suggest that changes in core cellular processes, like chlorophyll, amino acid, sugar, DNA, and reactive oxygen species (ROS) metabolism, are an outcome-or may be drivers—of plant ageing.

\section{Reactive oxygen species}

Well over half a century ago, Harman (1956) hypothesised that oxygen radicals, as by-products of endogenous metabolism, inflict oxidative stress in all living things, leading to loss of functional efficiency in multiple cellular processes and ultimately to ageing [24]. On top of metabolic processes present in all eukaryotes, photosynthetic activity in plants adds considerably to ROS production [25, 26]. At moderate levels, ROS help to modulate various growth and developmental processes [27], but an excess of ROS is toxic and causes damage to various cellular organelles and components [28, 29]. It is well reported that in plants, ageing is accompanied and accelerated by the overabundance of ROS [27, 30-33].

While relatively few transcriptomics reports have specifically addressed the ROS-ageing relationships, some of these studies indicate that cellular oxidative stress increases with plant and leaf age. For instance, leaves of 3000-yearold Platycladus orientalis had higher ROS levels together with the upregulation of genes involved in ROS production and stress responses than the leaves of a 20-year-old plant of the same species [34]. Likewise, age-dependent transcriptomes of Arabidopsis leaves showed that the expression of genes associated with oxidative stress were elevated in leaves before the completion of leaf growth and senescence initiation [6, 35]. An analysis of the Arabidopsis leaf transcriptome during ageing also showed enrichment of abiotic stress-responsive genes in matured leaves before the onset of senescence [19]. The upregulated oxidative stress genes include several senescence associated genes (SAGs) and genes that encode mitogen activated protein kinases (MAPKs), WRKY and NAC transcription factors, that function to limit oxidative stress. However, these studies did not differentiate between upregulation of genes solely to limit oxidative stress, or upregulation to control and allow an ageinduced increase in oxidative stress.

\section{Chlorophyll metabolism and photosynthesis}

Chlorophyll is an essential component of photosynthesis in plants and, therefore, it can be expected that biosynthesis of 
photosynthesis components is tightly coupled to leaf development and that such biosynthesis is ceased once senescence starts. However, since sugar status and the ability to export sugars out of the leaf can regulate leaf senescence, photosynthetic capacity may play a more direct role in the regulation of ageing and induction of leaf senescence [36-38]. Consistent with that idea, Breeze et al. found a significant switch in gene expression of photosynthesis-related genes, prior to the onset of senescence: reduced expression of two Rubisco small subunit genes that encode critical enzymes for $\mathrm{CO}_{2}$ fixation in the Calvin cycle was observed five days earlier than visual signs of leaf senescence [6]. Furthermore, the expression of genes involved in chlorophyll degradation, SGR1, SGR2, NYC1 (Chlorophyll b reductase) and $\mathrm{PaO}$ (Pheophorbide a oxygenase) increased before leaf tip yellowing was observed. Moreover, transcriptomic studies performed in Switchgrass cultivars showed that changes in expression of chlorophyll biosynthesis and degradationrelated genes occurred weeks before the visual onset of leaf senescence [39].

\section{Primary metabolites}

Biosynthesis of sugars and amino acids is tightly coordinated with photosynthesis to meet the plant's demand for energy required during development $[40,41]$ and must therefore be highly regulated. Sugars are the primary products of photosynthesis and are transported from source tissues to the sink organs via several sugar transporters [42]. In light, sugars are converted to starch, which is remobilised at night to support metabolism and growth of plants [43]. In a recent study, analysis of primary metabolites in progressively ageing Arabidopsis leaves showed that 14 sugar metabolites, including fructose, glucose, maltose, mannitol, galactose, trehalose, and other unidentified sugars that were high in young expanding leaves, decreased gradually during leaf expansion, and well before the onset of leaf senescence [35]. A similar pattern of reduction in sugar metabolites before the initiation of senescence was observed in comprehensive metabolomics studies performed on Nicotiana tabacum (tobacco) leaves during five developmental stages [44].

Several studies have found that sugar starvation due to limited photosynthesis activity initiates the onset of leaf senescence [37, 45-48] and gene expression involved in carbohydrate metabolism decreased before the onset of senescence [6]. Moreover, carbohydrate biosynthesis-related genes were upregulated in leaves during the growth to maturation stage but downregulated in the maturation to senescence stage [19].

Many primary metabolites have protective properties to help plants tolerate oxidative stress [49-52]. Crucially, the stress metabolites proline, putrescine, pyroglutamic acid, spermidine, citric acid, raffinose, and dehydroascorbic acid are well reported to play central roles in stress tolerance and were found to decrease during leaf maturation, before the onset of senescence (Fig. 1) [35, 44, 53]. Other basic metabolic changes that regulate senescence were reported in young and mature but not yet senescing leaves of both perennial and annual plants: for instance, downregulation of genes that are involved in amino acid biosynthesis and a decrease in the level of amino acids in developing Medicago sativa (alfalfa) and Arabidopsis leaves [6, 35, 54]. Likewise, in a recent study, age-related differences were observed in metabolites of leaves collected from perennial tea crop Camellia sinensis plants aged 8 and 25 years old [55]. Compared to 8-year-old samples, tea leaves collected from older plants showed lower alanine, glutamine, theanine, asparagine, leucine, succinate amino acid and sucrose, $\alpha$-glucose and $\beta$-glucose sugar abundances [55]. In this case, the leaves were of similar age and this suggests that primary metabolites may change not only with leaf age, but also with plant age in perennial plants. Thus, the age-related decrease of many primary metabolites may be a manifestation of leaf ageing processes, or may contribute to ageing.

\section{DNA repair}

Living organisms, including plants, are protected against DNA damage caused by endogenous and exogenous factors by means of DNA repair mechanisms [56, 57]. In plants, unrepaired DNA damage can lead to early onset of senescence [58]. Also, in humans, the accumulation of DNA damage over time is linked to early ageing $[59,60]$. Multiple studies suggest that accumulation of unrepaired DNA can gradually reduce cellular functions and accelerate ageing in a variety of species, including Saccharomyces cerevisiae, Caenorhabditis elegans, and Drosophila melanogaster [61-64]. Thus, there is evidence across kingdoms that links DNA damage with early ageing. In Arabidopsis, DNA repair efficiency was higher in young leaves than in adult leaves [65] and Arabidopsis transcriptomic data of expanding leaves also showed a strong negative relation between expression of genes involved in DNA repair mechanisms and age [35].

To further asses the possible role of DNA repair in ageing, we obtained a list of DNA repair-related genes from TAIR (https://www.Arabidopsis.org/) and visualised the gene expression patterns in Arabidopsis tissues from germination to the rosette bolting stage using the Genevestigator development tool (https://genevestigator.com/gv/; [66]). The heat map in Fig. 2 shows a very strong correlation between rosette development and expression of DNA repair genes, with the highest expression in young rosette tissues, and the lowest during the bolting stage. These results too indicate that DNA repair mechanisms are developmentally regulated, and therefore, maintenance of genomic integrity 
Fig. 1 Changes in metabolism in different aged leaves. Primary metabolic changes in young and adult leaf shown by transcriptomic and metabolomic studies. Green and red arrows indicate relative abundances of metabolites to be higher or lower, respectively, as compared between young and old leaves

\begin{tabular}{|c|c|c|c|}
\hline Amino acids & Young leaf & Adult leaf & References \\
\hline Alanine & 4 & $\downarrow$ & $35,44,54,55$ \\
\hline Asparagine & 4 & $\downarrow$ & $35,44,54,55$ \\
\hline Aspartic acid & 4 & $\downarrow$ & $35,44,54,55$ \\
\hline Glutamic acid & 4 & $\downarrow$ & 35,44 \\
\hline Glutamine & 4 & $\downarrow$ & $35,44,54,55$ \\
\hline Glycine & $\mathbf{4}$ & $\downarrow$ & 35,44 \\
\hline Lysine & 4 & $\downarrow$ & 35,44 \\
\hline Leucine & $\uparrow$ & $\downarrow$ & 55 \\
\hline Succinate & 4 & $\downarrow$ & 55 \\
\hline Hydroxyproline & $\boldsymbol{4}$ & $\downarrow$ & 35 \\
\hline Valine & $\boldsymbol{4}$ & $\downarrow$ & 35 \\
\hline Tyrosine & $\mathbf{4}$ & $\downarrow$ & 35 \\
\hline Theanine & 4 & $\downarrow$ & 54,55 \\
\hline$\alpha$-keto glutarate & 4 & $\downarrow$ & 53 \\
\hline \multicolumn{4}{|l|}{ Carbohydrates } \\
\hline Fructose & 4 & $\downarrow$ & 35 \\
\hline Fructose-6-phosphate & $\uparrow$ & $\downarrow$ & 44 \\
\hline Glucose, 1,6 -anhydro- $\beta$ & $\mathbf{4}$ & $\downarrow$ & 35 \\
\hline Glucose & $\mathbf{1}$ & $\downarrow$ & 35 \\
\hline$\alpha$-Glucose & $\mathbf{4}$ & $\downarrow$ & 54,55 \\
\hline$\beta$-Glucose & 4 & $\downarrow$ & 54,55 \\
\hline Glucose-6-phosphate & $\uparrow$ & $\downarrow$ & 44 \\
\hline Maltose & 4 & $\downarrow$ & 35 \\
\hline Mannitol & $\uparrow$ & $\downarrow$ & 35,44 \\
\hline Galactose & $\mathbf{4}$ & $\downarrow$ & 35,44 \\
\hline Trehalose & $\uparrow$ & $\downarrow$ & 35 \\
\hline Myo-Inositol & $\mathbf{4}$ & $\downarrow$ & 35,44 \\
\hline Sucrose & $\mathbf{4}$ & $\downarrow$ & $53,54,55$ \\
\hline Fucose & 4 & $\downarrow$ & 44 \\
\hline \multicolumn{4}{|l|}{$\begin{array}{c}\text { Stress protective } \\
\text { metabolites }\end{array}$} \\
\hline Citric acids & 4 & $\downarrow$ & 35,44 \\
\hline Dehydroascorbic acid & $\boldsymbol{4}$ & $\downarrow$ & 35 \\
\hline Malic acid & $\boldsymbol{4}$ & $\downarrow$ & 35 \\
\hline Proline & 4 & $\downarrow$ & $35,44,53$ \\
\hline Putrescine & $\boldsymbol{4}$ & $\downarrow$ & $35,44,53$ \\
\hline Pyroglutamic acid & 4 & $\downarrow$ & 35 \\
\hline Nicotinic acid & 4 & $\downarrow$ & 35,44 \\
\hline Raffinose & $\uparrow$ & $\downarrow$ & 44 \\
\hline Spermidine & 4 & $\downarrow$ & 35,44 \\
\hline Chlorophyll & 4 & $\downarrow$ & 6,39 \\
\hline DNA repair & 4 & $\downarrow$ & $35,65,66$ \\
\hline Gibberellic acid & 4 & $\downarrow$ & 35,69 \\
\hline Ethylene & $\downarrow$ & 4 & $19,34,35,69$ \\
\hline Abscisic acid & $\downarrow$ & 4 & $6,35,69$ \\
\hline Jasmonic acid & $\downarrow$ & 4 & $6,35,69$ \\
\hline Salicylic acid & $\downarrow$ & 4 & 35,69 \\
\hline Cellular ROS level & $\downarrow$ & 4 & $6,19,34,35$ \\
\hline
\end{tabular}


Fig. 2 Expression patterns of DNA repair-related genes, obtained from TAIR, in Arabidopsis tissue of increasing age, plotted by the Genevestigator development tool. Wild type Arabidopsis mRNA dataset was chosen to generate the heat map. In Genevestigator, the colours (values) are normalised to the expression potential of each gene and the expression potential indicated for a given stage of development is the average of expression of all samples annotated. The colour scale bar from white to blue shows the percentage of the gene expression potential. The darkest blue represents the maximum level of expression and white colour represents the minimum level of expression
$0 \%$

$100 \%$

Percent of expression potential

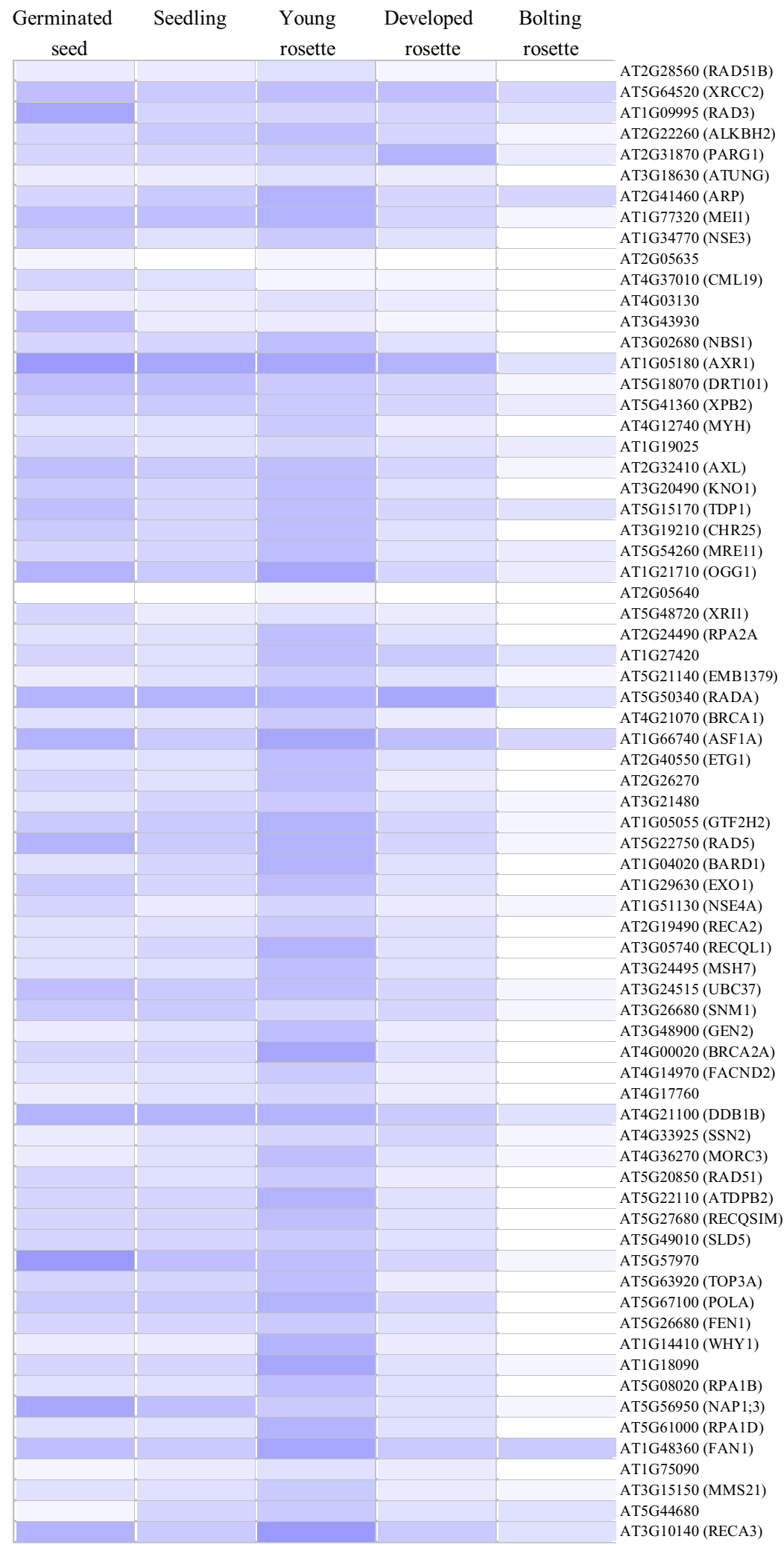


may decrease as the leaves age. A confounding factor is that during leaf development, the cell cycle completes, resulting in a reduced requirement for DNA repair mechanisms [65]. Nevertheless, DNA damage will still occur and the combination of increased oxidative stress during leaf expansion [35] and reduced DNA repair may result in decreased genome stability and ageing. Moreover, these results are solely based on Arabidopsis tissues.

\section{Hormones}

Plant hormones have a wide range of functions during all phases of development and thus also during ageing. The hormones ethylene, abscisic acid (ABA), jasmonic acid (JA), and salicylic acid (SA) have been described to initiate plant ageing, while cytokinin, auxin, and gibberellic acid (GA) are believed to delay plant ageing [17, 67, 68]. Arabidopsis leaf transcriptome analysis is in general agreement with this $[6$, $35,69]$, although a more or less equal percentage of cytokinin and auxin-related genes were up and downregulated in expanding leaves [35, 69]. Fifteen out of 18 GA biosynthesis-related genes decreased in Arabidopsis leaves from the expanding to the senescence phase, which suggests that active forms of GA decline as the leaf ages [69]. Ethylene is known as a master regulatory hormone in inducing leaf senescence [70-72]. Chang et al. showed that the expression of ethylene biosynthesis-related genes was increased in an old Platycladus orientalis tree as compared to a young one [34], and in Arabidopsis, all the ethylene-responsive genes were upregulated in mature leaves as compared to young leaves [35]. Likewise, during late developmental stages, i.e., from maturation to senescence, Arabidopsis leaf transcriptomes showed the enrichment of genes associated with ethylene biosynthesis and signalling [19, 69]. Moreover, the majority of genes involved in the biosynthesis and signalling of ethylene, SA, ABA, and JA, increased during Arabidopsis leaf expansion [35]. Also, the expression of a vast majority of JA, ABA, and SA biosynthesis-related genes increased in Arabidopsis from the rapid expansion leaf stage to the leaf senescence stage, suggesting a role for these hormones in leaf ageing [69]. Thus, leaf transcriptome findings support the well-known roles of ethylene, SA, ABA, and JA in ageing.

\section{Changes in metabolic signatures during leaf ageing}

Transcriptomic and metabolomic studies of developing leaves show a strong correlation between core cellular processes and ageing (Figs. 1 and 3). During early leaf development, core cellular processes like chlorophyll biosynthesis and DNA repair accompany growth, while the abundance of sugars, amino acids, GA, and metabolites that provide stress tolerance, deliver the means to sustain active growth.
Then, as the leaf matures, an increase in cellular oxidative stress and senescence-inducing hormones coincides with a marked reduction in chlorophyll biosynthesis, DNA repair and abundance of sugars, amino acids, and stress-tolerant metabolic activities. Thus, Fig. 3 illustrates the patterns of core cellular processes in young and adult leaves, underpinning their potential role as major age-regulatory factors.

However, the transcriptomic and metabolomic work unveiled correlative, rather than causal relationships. For example, these studies do not distinguish between the hypotheses that chlorophyll biosynthesis, and the drop thereof, drives ageing or, that ageing processes drive chlorophyll anabolic and catabolic processes. Fortunately, ample molecular genetic studies, dating back decades and more current ones, have shown causality. Below we aim to test hypotheses suggested by above-described work using mutant and physiological studies that perturb primary metabolism pathways.

\section{Molecular genetic approaches confirm the role of core cellular processes in leaf ageing}

Gene knock-out and overexpression studies are extremely powerful at demonstrating causal relationships and may provide the best proof for pinpointing genes and processes involved in the regulation of ageing. Nevertheless, knockout of essential genes involved in development or primary metabolism can render a plant unviable, and leaky mutations may not exhibit a clear phenotype. Moreover, genes that possess multiple functions, which is often the case for those involved in hormone biosynthesis and response, may affect plants during early growth, and this in turn may cause secondary developmental changes that wrongly connect the gene knock-out to the regulation of ageing. Overexpression of genes can cause ectopic phenotypes, which may mask or alter an ageing phenotype. Conditional knock-down or overexpression studies may solve these issues, but it is difficult to create complete knock-outs at the right time and tissue. Therefore, to conclude that a metabolic pathway regulates ageing, multiple studies-preferably using various gene perturbations in the process-should be performed. Below we describe combined molecular genetic evidence in core cellular processes to conclude whether they regulate, or correlate with ageing.

\section{ROS metabolism drives ageing}

Since ROS play important roles in signalling and their abundance is well controlled using multiple scavenging pathways [29, 73, 74], a direct correlation between ROS and ageing has been difficult to establish. Nevertheless, 
Fig. 3 Core cellular processes contribute to plant ageing. A combined systems biology and molecular genetic approach suggests that changes in core cellular processes that occur during leaf ageing are regulating ageing. The model shows that the elevated growthpromoting metabolites in young leaves decrease in mature and old leaves, whereas the lower levels of cellular oxidative stress and stress-responsive hormones elevate as the leaf ages. $G A$ gibberellic acid, $A B A$ abscisic acid, $J A$ jasmonic acid, $S A$ salicylic acid

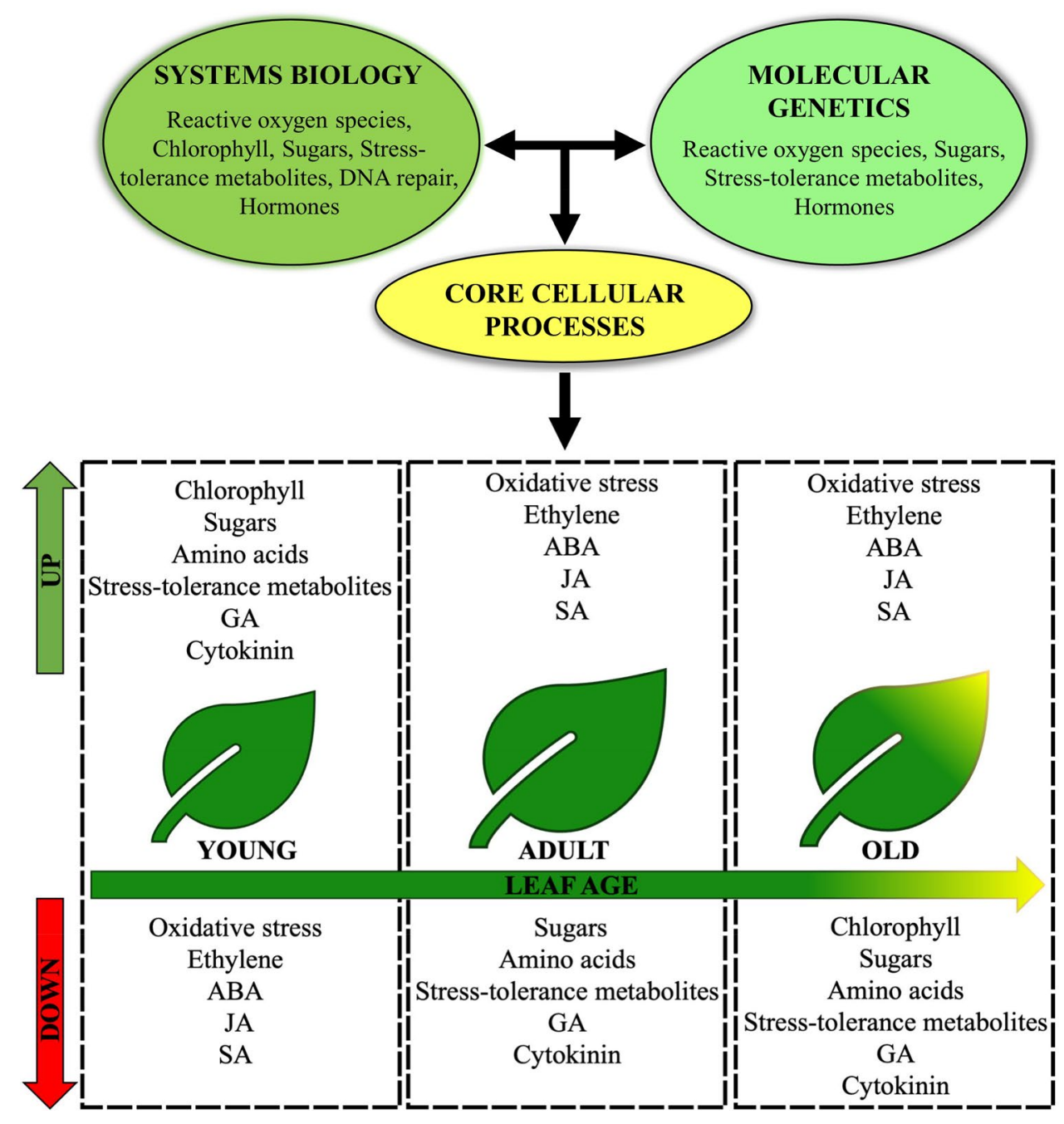

a good number of studies in the model plants Arabidopsis and Oryza sativa (rice) have helped to understand the involvement of ROS in plant ageing. Woo et al. showed that oxidative stress tolerance is associated with leaf longevity in the Arabidopsis oresara mutants: orel (NAC Transcription factor), ore3 (EIN2) and ore9 (F-Box protein) [75]. However, the reason for the oxidative stress tolerance in those mutants is elusive as those mutants did not show increased activity of antioxidants compared to wild type. The onset of leaf death5 (old5) early ageing Arabidopsis mutant showed a higher respiration rate, along with higher oxidative stress, well before the onset of senescence [76]. Single and double mutants of cat2, cat3, and cat $2 /$ cat 3 did not show any significant differences during development, with not much reduction in ROS accumulation [77]. This suggests that there are other ROS scavenging channels that compensate the ROS detoxification in the absence of catalases in those mutant plants. A wellstudied ROS regulatory gene encodes the $\mathrm{H}_{2} \mathrm{O}_{2}$-induced
NAC Transcription factor (JUB1), which functions to reduce $\mathrm{H}_{2} \mathrm{O}_{2}$ production [78-81]. Overexpressed lines of JUB1 in Arabidopsis had a diminished intracellular $\mathrm{H}_{2} \mathrm{O}_{2}$ production, thereby delaying bolting and plant ageing. In contrast, the jub1-1 knock-out mutant showed early bolting and early ageing with lower tolerance to abiotic stress [79]. In rice, inactivation of UDP-N-acetylglucosamine pyrophosphorylase 1 (OsUAP1), Nicotinate Phosphoribosyl transferase (OsNaPRT1), O-methyltransferase (OsMTS1), reactive oxygen species-sensitive leaf senescencel (RLS1), and premature senescence of flag leaf ( $p s f$, encoding superoxide dismutase), all showed early leaf ageing as evidenced by premature induction of a leaf senescence phenotype, that correlated with an increase in cellular ROS, hypersensitivity to oxidative stress, or a lower activity of ROS-scavenging enzymes [82-86].

Thus, consistent with the transcriptomic studies, considerable genetic evidence corroborates the relationship between ROS and plant ageing. 


\section{Chlorophyll metabolism can affect senescence}

Photosynthesis captures energy and transforms it into sugars and in the process produces ROS [73, 87]. Thus, photosynthesis could contribute to regulating ageing in different ways. A reduction in chlorophyll biosynthesis may decrease the ability of an ageing cell to maintain its chloroplasts and therefore decrease photo assimilate production. In addition, photosynthesis-related ROS production may contribute to ageing and the degradation of chlorophyll involves the production of highly phototoxic intermediate degradation products [88].

Arabidopsis, rice and Medicago truncatula mutants that stay green for longer than the wild type have been isolated, thus suggesting that ageing and senescence may be delayed. Some of these mutations were found to affect chlorophyll degradation, for example, pph -1 (pheophytinase), nyc 1 (chlorophyll b reductase or non-yellow coloring1), sgr (staygreen) and nol (NYC1-LIKE) [89-97]. However, in these mutants, photosynthetic activity was not altered, and cellular damage not delayed as compared to the wild type. Thus, such cosmetic (non-functional) mutants are useful for the study of chlorophyll degradation but not for studying leaf ageing, as the retention of greenness in these mutants does not lead to increased leaf longevity.

However, transcription factors ANAC072, ANAC046 and ANAC016 were found to regulate chlorophyll degradation and senescence by binding to the promoter sites of NONYELLOWING1 (NYE1, also known as SGR1) and activating the transcription of these genes [98-100]. The loss of function mutant anac072-1 showed a staygreen phenotype with slower retardation of photosynthetic efficiency than wild type, whereas the overexpression mutant anac072-2 exhibited an opposite phenotype [98]. Furthermore, various staygreen mutants of durum wheat maintained photosynthetic competence for longer than the control plant [101]. Thus, these studies show that some genes that regulate chlorophyll degradation, regulate leaf senescence as well. However, there is little evidence for a role of chlorophyll metabolism in ageing processes other than senescence.

\section{Primary metabolism affects ageing}

Sugars play critical roles during all aspects of plant growth $[102,103]$ and mutations that cause altered sugar metabolism are likely to affect aspects of plant growth, including ageing, making it difficult to pinpoint a direct effect of sugars on ageing. Nevertheless, in Arabidopsis, sugar signalling has been shown to affect an important ageing switch in Arabidopsis, the transition from juvenile to adult phase [104]. A lower sugar content led to increased abundance of microRNA156 (miR156), thereby restricting the transition of juvenile leaves to adult leaves, while an exogenous supply of sucrose promoted adult leaf growth by repressing the transcription of miR156 genes [104, 105]. Sugars were also convincingly demonstrated to affect another aspect of ageing: the timing of leaf senescence. Lower sugar abundances generally delay senescence. In artificial media, high glucose in combination with low nitrogen can induce senescence [37, 47]. Wang et al. showed that in the rice premature leaf senescence 2 ( $p l s 2$ ) mutant accumulation of sucrose and starch grains, in chloroplast containing cells, reduced expression of photosynthesis-related genes in combination with premature leaf senescence [106]. Disruptions of genes that sense or regulate sugars also affect the timing of senescence. Mutations in Arabidopsis Hexokinase-1 (HXK-1) decreased hexose abundance and delayed leaf senescence [47, 107], while its overexpression in Solanum lycopersicum (tomato) induced leaf senescence [108]. Furthermore, an orthologue of sucrose nonfermenting1 (SNF1)/Snfl-related kinase1 (SnRK1) acts as a sensor for starvation or low glucose condition [109]. Transgenic Arabidopsis with overexpressed KIN10 (SnRK1 homologue) showed longer leaf longevity and increased tolerance to nutrient deficiency. Likewise, early leaf senescence was reported in plants with reduced levels of KINIO and KINII [110]. Other studies, some of which used dark-detached leaves, showed that sugar starvation may induce leaf senescence and external application of sugars can retard the senescence process [48, 111-113]. Thus, while sugars regulate ageing, the molecular basis of this mechanism is poorly understood.

Other metabolites have also been linked to plant ageing, especially those that are involved in ROS and stress regulation. The polyamines putrescine, spermidine, and spermine and $\gamma$-amino butyric acid (GABA), are all associated with the regulation of ageing in plants. In Arabidopsis, the GABA transaminase knock-out mutant (pop2) showed higher ROS accumulation, early flowering and early onset of leaf senescence [114]. Constitutive overexpression of arginine decarboxylase $(A D C)$ and polyamine uptake transporters (PUT) caused accumulation of putrescine and spermidine, respectively, in leaves and displayed a delayed flowering phenotype $[115,116]$. Furthermore, the Polyamine Oxidase 4 (pao4) mutant had increased spermine and reduced spermidine contents, and showed a delayed onset of dark-induced leaf senescence. The pao4 mutant was also associated with reduced $\mathrm{H}_{2} \mathrm{O}_{2}$ and lipid peroxidation levels and increased abundance of redox regulating metabolites [117]. In rice, the spotted leaf32 (spl32) mutation disrupted the gene encoding ferredoxin-dependent glutamate synthase (Fd-GOGAT), which is involved in biosynthesis of glutamate. This mutant showed lower glutamate accumulation combined with higher levels of $\mathrm{H}_{2} \mathrm{O}_{2}$ and other major amino acids and these changes coincided with early leaf senescence [118]. 
These studies suggest that changes in contents of many primary metabolites regulate ageing, some of those likely by means of altering ROS levels.

\section{Do DNA repair processes regulate ageing?}

Transcriptome analyses provide a strong argument that DNA repair mechanisms are regulating ageing in plants. Also, based on extensive research in other eukaryotes, decreased DNA repair can be expected to limit the lifespan of plants. However, little firm evidence confirms this hypothesis. Two decades ago, Riha et al. studied Arabidopsis telomerasedeficient T-DNA lines of AtTERT and while early knockout generations appeared phenotypically normal, later generations displayed massive chromosomal instability [119]. The loss of telomeres impaired growth, but the leaves of the mutant remained green and metabolically active for months. It was also shown that Arabidopsis ddml (decrease in DNA methylation 1) mutant plants exhibited delayed ageing with reduced DNA repair capabilities and susceptibility to salt stress [120,121]. These studies support a link between DNA repair and ageing in plants. On the contrary, mutations in the rad51b, rad5ld, and $x r c c 2$ genes, which are important for DNA repair displayed normal vegetative and reproductive growth [122], and we did not detect an ageing phenotype in the recQ4A mutant that displayed genomic instability, despite actively looking for it [123].

Recently, a comprehensive study performed by Li et al., separately knocked out 13 Arabidopsis genes involved in DNA repair and specifically looked for leaf ageing phenotypes. Surprisingly, only ATM (Ataxia Telangiectasia Mutated) protein deficiency caused early leaf ageing, resulting from an over-accumulation of double-strand breaks [124]. This raises the question: does DNA repair-redundancy in plants mitigate the effect of single gene knock-outs, or does the ATM mutant affect ageing as a secondary result of gross genome instability? These studies indicate that DNA repair is not a major player in Arabidopsis ageing regulation. Nevertheless, the relationship between DNA repair and plant ageing may be complex and could differ between plant species; thus, further study into this area is warranted.

\section{Hormones are major plant growth regulators and govern ageing}

Plant hormones are one of the most important internal factors that regulate plant development and influence ageing. Over the past few decades, hormones have been studied extensively, and excellent reviews have been published on the role of hormones that positively or negatively regulate plant ageing [17, 125-129]. There is ample genetic evidence confirming the role of ethylene, JA, SA and ABA as positive and GAs as negative regulators of ageing [17, 130-132].
However, the role of auxin in plant ageing is less understood and contradictory. Some reports describe auxin as a promoter of ageing, whereas others consider it to delay ageing. For instance, abundance of the biologically active auxin hormone indoleacetic acid (IAA) was found to increase during age-dependent leaf senescence [133], suggestive of a role in senescence induction. Auxin can also induce the biosynthesis of ethylene, which is a strong senescence-inducing hormone [134]. Furthermore, a mutation in the SAUR36 gene, which mediates auxin-induced leaf senescence, showed delayed plant ageing [135]. Likewise, mutations in Arabidopsis ARF1 and ARF2 (Auxin response factor) delayed leaf senescence progression in the mutant plants [136, 137]. However, treatment of Arabidopsis leaves with auxin decreased expression of senescence marker gene SAG12 [138], and 35S:YUC6 transgenic plants, which exhibit increased IAA abundance, showed extended leaf longevity [139]. Thus, a dual role of auxin has been observed in plants' ageing, which does not contradict the transcriptomic studies.

Transcriptomic studies do not provide clear hypotheses as to whether cytokinins are positive or negative regulators of ageing. However, molecular genetic analyses clearly demonstrate that the cytokinin hormone delays ageing in plants. For example, various transgenic plants introduced with the IPT gene (Isopentyl transferase, a gene involved in cytokinin biosynthesis) under various promoters showed delayed senescence [140-143]. Also, the cytokinin level in old tobacco leaves was lower than that in young leaves [144]. Furthermore, the exogenous application of cytokinin on numerous monocot and dicots plants is reported to delay senescence by preventing chlorophyll breakdown and inhibiting protein degradation [127, 144-146].

Thus, functions of major plant hormones in regulating plant ageing as predicted by transcriptomics studies, can be largely confirmed by molecular genetic experiments, although exceptions, notably the roles of auxins and cytokinin, exist.

\section{Primary metabolism as drivers of ageing}

Understanding ageing - and ultimately being able to control it-is one of the holy grails of biological sciences. Nevertheless, progress in this area is painfully slow, and past molecular genetic analyses have provided important, but sometimes confusing results. Recent advances in systems biology have allowed us to develop new, testable hypotheses and, crucially, many of these have already been tested. A picture is emerging where basic biological processes are master controllers of ageing (Fig. 3). In plants, hormones have long been demonstrated to play important roles in ageing and this view has not changed, with ethylene, ABA, JA, SA as 


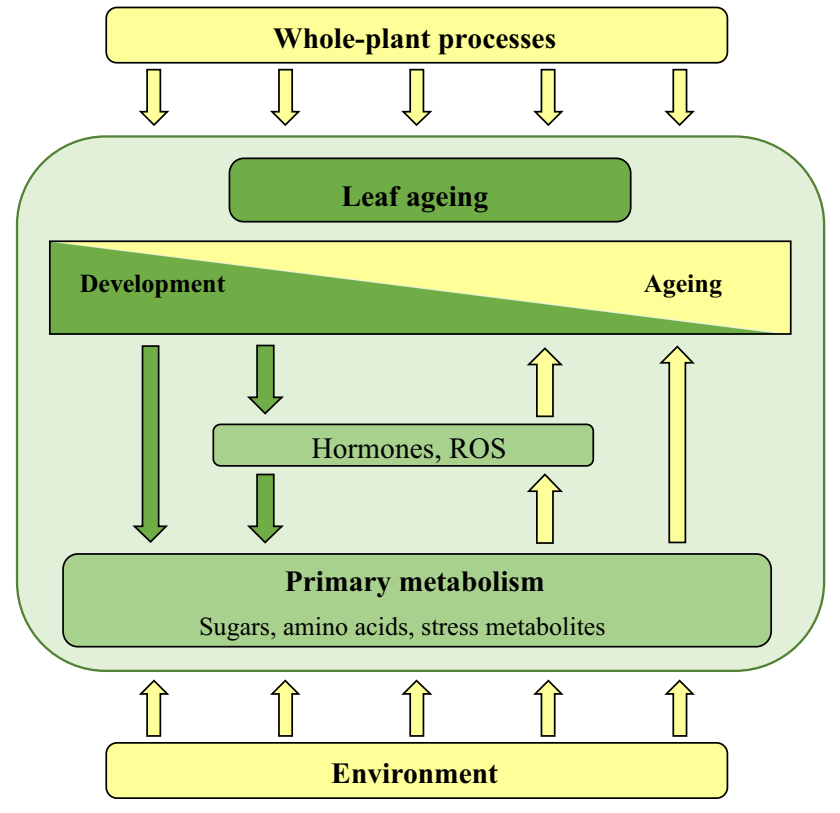

Fig. 4 Speculative model proposing that leaf development affects primary metabolism and that this inevitably leads to leaf ageing. Plant hormones and reactive oxygen species (ROS) play a central role in this process as they are affected by development and primary metabolites. The speed of ageing, but not leaf ageing itself, can be modulated by environmental stress and whole-plant processes, such as reproduction in monocarpic and season in deciduous plants

ageing-inducing hormones and GA and cytokinin as ageing repressors. Nevertheless, all plant hormones fulfil multiple roles throughout plant development, which may explain discrepancies between predictions based on transcriptomics and molecular genetic analyses. The systems biology approaches furthermore strongly support a role for oxidative stress in ageing. In addition, core cellular processes appear to be fundamental to ageing, possibly feeding into oxidative stress metabolism (Fig. 3).

Expanding on this idea, we propose that changes in primary metabolite abundances, caused by leaf developmental processes, are driving leaf ageing. The concept that leaf development is intimately linked with leaf ageing is not new, but here we posit the idea that primary metabolism constitutes this link (Fig. 4). The figure highlights that leaf development inherently affects primary metabolism, directly and through the action of hormones and ROS. For example, the end of cell division and expansion, changing source-sink partitioning and a decrease in photosynthetic efficiency changes the abundances of sugars and ROS; decreased metabolites contributing to antioxidant activity further increases ROS abundance. These changes in primary metabolites then drive ageing, likely at least partly through modulation of ROS abundance. The figure also shows that leaf ageing is not a rigid process: environmental factors like stress and whole-plant processes such as monocarpic senescence can modulate ageing of individual leaves. The model thus hypothesises how leaf ageing is hard-wired to developmental processes and is consistent with decades-long research, where no mutant has been found that does not age, and many ageing mutants show alterations in development.

Plant ageing is still far from being understood. What can be done to move this field forwards? Since ageing is so closely integrated into development-or perhaps they can even be considered one and the same-it will be difficult to separate developmental from ageing processes. Nevertheless, the model presented in Fig. 4 makes two predictions that may help design experiments that can test the model: (1) ageing is a continuous process and if early ageing is affected, then the timing of the final leaf ageing stage, leaf senescence, should also be affected; (2) primary metabolism affects ageing throughout leaf development. Most research in plant ageing has been done on senescence, which is the final stage of ageing. Therefore, increased emphasis should be placed on the early stages of ageing, while the onset of leaf senescence could still be used to estimate total leaf longevity. Furthermore, transient disruptions in primary metabolism may not affect whole plant development, while the model predicts that this would affect leaf ageing and lifespan. Here, multiomics approaches, including metabolomics, can detect patterns that may not be detected using targeted analyses. It may be more challenging to study the early developmental stages because they are less defined, and many growth processes take place at the same time. However, systems biology analyses can tease out individual processes and molecular genetic approaches can robustly test hypotheses. We also would like to encourage researchers to test the model in diverse plant species, including perennials, to determine how distinct plant species modulate general ageing processes to benefit survival. We are looking forward to further progress in this age-old scientific field.

Acknowledgements The authors would like to acknowledge the published work of many researchers whose work has relevance for this review, but were not cited because of space and our own limitations. A.K. acknowledges the European Union's Horizon 2020 research and innovation programme, project PlantaSYST (SGA-CSA No. 739582 under FPA No. 664620), as well as the BG05M2OP001-1.003-001-C01 project, financed by the European Regional Development Fund through the Bulgarian 'Science and Education for Smart Growth' Operational Programme.

Author contributions AK performed the Genevestigator analysis and metabolic analysis. All authors wrote and approved the manuscript.

Funding No specific funding was obtained for this manuscript.

Data availability All data generated and analysed during this study are included in this manuscript. 


\section{Declarations}

Conflict of interest The authors declare that they have no competing interests.

Ethics approval and consent to participate Not applicable.

Consent for publication Not applicable.

Open Access This article is licensed under a Creative Commons Attribution 4.0 International License, which permits use, sharing, adaptation, distribution and reproduction in any medium or format, as long as you give appropriate credit to the original author(s) and the source, provide a link to the Creative Commons licence, and indicate if changes were made. The images or other third party material in this article are included in the article's Creative Commons licence, unless indicated otherwise in a credit line to the material. If material is not included in the article's Creative Commons licence and your intended use is not permitted by statutory regulation or exceeds the permitted use, you will need to obtain permission directly from the copyright holder. To view a copy of this licence, visit http://creativecommons.org/licenses/by/4.0/.

\section{References}

1. Lanner RM, Connor KF (2001) Does bristlecone pine senesce? Exp Gerontol 36:675-685

2. Arnaud-Haond S, Duarte CM, Diaz-Almela E, Marbà N, Sintes T, Serrão EA (2012) Implications of extreme life span in clonal organisms: millenary clones in meadows of the threatened seagrass Posidonia oceanica. PLoS ONE 7(2):e30454

3. Dijkwel PP, Lai AG (2019) Hypothesis: plant stem cells hold the key to extreme longevity. Transl Med Aging 3:14-16

4. Sheldrake AR (1974) The ageing, growth and death of cells. Nature 250:381-385

5. Woo HR, Masclaux-Daubresse C, Lim PO (2018) Plant senescence: how plants know when and how to die. J Exp Bot 69:715-718

6. Breeze E, Harrison E, McHattie S et al (2011) High-resolution temporal profiling of transcripts during Arabidopsis leaf senescence reveals a distinct chronology of processes and regulation. Plant Cell 23:873-894

7. Thomas H (2013) Senescence, ageing and death of the whole plant. New Phytol 197:696-711

8. Lim PO, Kim HJ, Nam HG (2007) Leaf senescence. Annu Rev Plant Biol 58:115-136

9. Mattila H, Valev D, Havurinne V et al (2018) Degradation of chlorophyll and synthesis of flavonols during autumn senescence-the story told by individual leaves. AoB Plants. https:// doi.org/10.1093/aobpla/ply028

10. Bajji M, Kinet JM, Lutts S (2002) The use of the electrolyte leakage method for assessing cell membrane stability as a water stress tolerance test in durum wheat. Plant Growth Regul 36:61-70

11. Hörtensteiner S (2009) Stay-green regulates chlorophyll and chlorophyll-binding protein degradation during senescence. Trends Plant Sci 14:155-162

12. Buchanan-Wollaston V (1997) The molecular biology of leaf senescence. J Exp Bot 48:181-199

13. Diaz C, Lemaitre T, Christ A et al (2008) Nitrogen recycling and remobilization are differentially controlled by leaf senescence and development stage in Arabidopsis under low nitrogen nutrition. Plant Physiol 147:1437-1449
14. Guiboileau A, Sormani R, Meyer C, Masclaux-Daubresse C (2010) Senescence and death of plant organs: nutrient recycling and developmental regulation. CR Biol 333:382-391

15. Hortensteiner S (2002) Nitrogen metabolism and remobilization during senescence. J Exp Bot 53:927-937

16. Kim J, Kim JH, Il LJ, Woo HR, Lim PO (2018) New insights into the regulation of leaf senescence in Arabidopsis. J Exp Bot 69:787-799

17. Jibran R, Hunter DA, Dijkwel PP (2013) Hormonal regulation of leaf senescence through integration of developmental and stress signals. Plant Mol Biol 82:547-561

18. Kim J, Woo HRR, Nam HGG (2016) Toward systems understanding of leaf senescence: an integrated multi-omics perspective on leaf senescence research. Mol Plant 9:813-825

19. Woo HR, Koo HJ, Kim J et al (2016) Programming of plant leaf senescence with temporal and inter-organellar coordination of transcriptome in Arabidopsis. Plant Physiol 171:452-467

20. Penfold CA, Buchanan-Wollaston V (2014) Modelling transcriptional networks in leaf senescence. J Exp Bot 65:3859-3873

21. Cavill R, Jennen D, Kleinjans J, Briedé JJ (2016) Transcriptomic and metabolomic data integration. Brief Bioinform 17:891-901

22. Zhang $\mathrm{L}, \mathrm{Ma} \mathrm{C}$, Chao $\mathrm{H}$ et al (2019) Integration of metabolome and transcriptome reveals flavonoid accumulation in the intergeneric hybrid between Brassica rapa and Raphanus sativus. Sci Rep 9:1-8

23. Kogel KH, Voll LM, Schäfer P et al (2010) Transcriptome and metabolome profiling of field-grown transgenic barley lack induced differences but show cultivar-specific variances. PNAS 107:6198-6203

24. Harman D (1956) Aging: a theory based on free radical and radiation chemistry. J Gerontol 11:298-300

25. Navari-Izzo F, Pinzino C, Quartacci MF, Sgherri CLM (1999) Superoxide and hydroxyl radical generation, and superoxide dismutase in PSII membrane fragments from wheat. Free Radic Res 31:3-9

26. Karuppanapandian T, Moon JC, Kim C, Manoharan K, Kim W (2011) Reactive oxygen species in plants: their generation, signal transduction, and scavenging mechanisms. Aust J Crop Sci 5:709-725

27. Mhamdi A, Van Breusegem F (2018) Reactive oxygen species in plant development. Development 145:dev164376

28. Sewelam N, Kazan K, Schenk PM (2016) Global plant stress signaling: reactive oxygen species at the cross-road. Front Plant Sci 7:187

29. Huang H, Ullah F, Zhou DX, Yi M, Zhao Y (2019) Mechanisms of ROS regulation of plant development and stress responses. Front Plant Sci 10:800

30. Zimmermann P, Zentgraf U (2005) The correlation between oxidative stress and leaf senescence during plant development. Cell Mol Biol Lett 10:515-534

31. Mayta ML, Lodeyro AF, Guiamet JJ et al (2018) Expression of a plastid-targeted flavodoxin decreases chloroplast reactive oxygen species accumulation and delays senescence in aging tobacco leaves. Front Plant Sci 9:1039

32. Wei T, Wang Y, Xie Z et al (2019) Enhanced ROS scavenging and sugar accumulation contribute to drought tolerance of naturally occurring autotetraploids in Poncirus trifoliata. Plant Biotechnol J 17:1394-1407

33. Kanojia A, Dijkwel PP (2018) Abiotic stress responses are governed by reactive oxygen species and age. Annu Plant Rev $1: 295-326$

34. Chang E, Zhang J, Deng N et al (2017) Transcriptome differences between 20- and 3,000-year-old Platycladus orientalis reveal that ROS are involved in senescence regulation. Electron J Biotechnol 29:68-77 
35. Kanojia A, Gupta S, Benina M et al (2020) Developmentally controlled changes during Arabidopsis leaf development indicate causes for loss of stress tolerance with age. J Exp Bot 71:6340-6354

36. Lara MEB, Garcia MCG, Fatima T et al (2004) Extracellular invertase is an essential component of cytokinin-mediated delay of senescence. Plant Cell 16:1276-1287

37. Wingler A, Marès M, Pourtau N (2004) Spatial patterns and metabolic regulation of photosynthetic parameters during leaf senescence. New Phytol 161:781-789

38. Wingler A (2018) Transitioning to the next phase: The role of sugar signaling throughout the plant life cycle. Plant Physiol 176:1075-1084

39. Palmer NA, Chowda-Reddy RV, Muhle AA et al (2019) Transcriptome divergence during leaf development in two contrasting switchgrass (Panicum virgatum $\mathrm{L}$.) cultivars. PLoS ONE 14:e222080

40. Galili G, Avin-Wittenberg T, Angelovici R, Fernie AR (2014) The role of photosynthesis and amino acid metabolism in the energy status during seed development. Front Plant Sci 5:447

41. Häusler RE, Heinrichs L, Schmitz J, Flügge UI (2014) How sugars might coordinate chloroplast and nuclear gene expression during acclimation to high light intensities. Mol Plant 7:1121-1137

42. Chen LQ (2014) SWEET sugar transporters for phloem transport and pathogen nutrition. New Phytol 201:1150-1155

43. Zeeman SC, Kossmann J, Smith AM (2010) Starch: its metabolism, evolution, and biotechnological modification in plants. Annu Rev Plant Biol 61:209-234

44. Li L, Zhao J, Zhao Y et al (2016) Comprehensive investigation of tobacco leaves during natural early senescence via multiplatform metabolomics analyses. Sci Rep 6:37976

45. Morkunas I, Borek S, Formela M, Ratajczak L (2012) Plant responses to sugar starvation carbohydrates-comprehensive studies on glycobiology and glycotechnology. IntechOpen, London, pp 409-438

46. Tetley RM, Thimann KV (1974) The metabolism of oat leaves during senescence: I. Respiration, carbohydrate metabolism, and the action of cytokinins. Plant Physiol 54:294-303

47. Pourtau N, Jennings R, Pelzer E, Pallas J, Wingler A (2006) Effect of sugar-induced senescence on gene expression and implications for the regulation of senescence in Arabidopsis. Planta 224:556-568

48. Zhaowei L, Qian Z, Fangmin C (2020) Sugar starvation enhances leaf senescence and genes involved in sugar signaling pathways regulate early leaf senescence in mutant rice. Rice Sci 27:201-214

49. Elsayed AI, Rafudeen MS, Golldack D (2014) Physiological aspects of raffinose family oligosaccharides in plants: protection against abiotic stress. Plant Biol 16:1-8

50. Nishizawa-Yokoi A, Yabuta Y, Shigeoka S (2008) The contribution of carbohydrates including raffinose family oligosaccharides and sugar alcohols to protection of plant cells from oxidative damage. Plant Signal Behav 3:1016-1018

51. Li Z, Yu J, Peng Y, Huang B (2016) Metabolic pathways regulated by $\gamma$-aminobutyric acid (GABA) contributing to heat tolerance in creeping bentgrass (Agrostis stolonifera). Sci Rep 6:30338

52. Mallhi ZI, Rizwan M, Mansha A et al (2019) Citric acid enhances plant growth, photosynthesis, and phytoextraction of lead by alleviating the oxidative stress in castor beans. Plants $8: 525$

53. Li W, Zhang H, Li X et al (2017) Integrative metabolomic and transcriptomic analyses unveil nutrient remobilization events in leaf senescence of tobacco. Sci Rep 7:12126
54. Yuan J, Sun X, Guo T, Chao Y, Han L (2020) Global transcriptome analysis of alfalfa reveals six key biological processes of senescent leaves. PeerJ 8:e8426

55. Mozumder NHMR, Lee YR, Hwang KH, Lee MS, Kim EH, Hong YS (2020) Characterization of tea leaf metabolites dependent on tea (Camellia sinensis) plant age through 1H NMR-based metabolomics. Appl Biol Chem 63:10

56. Karthika V, Babitha KC, Kiranmai K, Shankar AG, Vemanna RS, Udayakumar M (2020) Involvement of DNA mismatch repair systems to create genetic diversity in plants for speed breeding programs. Plant Physiol Rep 25:185-199

57. Singh SK, Roy S, Choudhury SR, Sengupta DN (2010) DNA repair and recombination in higher plants: insights from comparative genomics of Arabidopsis and rice. BMC Genom 11:443

58. Britt AB (1996) DNA damage and repair in plants. Annu Rev Plant Physiol Plant Mol Biol 47:75-100

59. Menck CFM, Munford V (2014) DNA repair diseases: what do they tell us about cancer and aging? Genet Mol Biol 37:220-233

60. Schumacher B, Garinis GA, Hoeijmakers JHJ (2008) Age to survive: DNA damage and aging. Trends Genet 24:77-85

61. Garschall K, Dellago H, Gáliková M, Schosserer M, Flatt T, Grillari J (2017) Ubiquitous overexpression of the DNA repair factor dPrp19 reduces DNA damage and extends Drosophila life span. NPJ Aging Mech Dis 3:5

62. Hyun M, Lee J, Lee K, May A, Bohr VA, Ahn B (2008) Longevity and resistance to stress correlate with DNA repair capacity in Caenorhabditis elegans. Nucleic Acids Res 36:1380-1389

63. Lombard DB, Chua KF, Mostoslavsky R, Franco S, Gostissa M, Alt FW (2005) DNA repair, genome stability, and aging. Cell 120:497-512

64. Madia F, Gattazzo C, Fabrizio P, Longo VD (2007) A simple model system for age-dependent DNA damage and cancer. Mech Ageing Dev 128:45-49

65. Golubov A, Yao Y, Maheshwari P et al (2010) Microsatellite instability in Arabidopsis increases with plant development. Plant Physiol 154:1415-1427

66. Hruz T, Laule O, Szabo G et al (2008) Genevestigator V3: a reference expression database for the meta-analysis of transcriptomes. Adv Bioinform 2008:420747

67. Xiong L, Zhu J-K (2003) Regulation of abscisic acid biosynthesis. Plant Physiol 133:29-36

68. Glazebrook J (2001) Genes controlling expression of defense responses in Arabidopsis-2001 status. Curr Opin Plant Biol 4:301-308

69. Van Der Graaff E, Schwacke R, Schneider A, Desimone M, Flügge UI, Kunze R (2006) Transcription analysis of Arabidopsis membrane transporters and hormone pathways during developmental and induced leaf senescence. Plant Physiol 141:776-792

70. Jing HC, Schippers JHM, Hille J, Dijkwel PP (2005) Ethyleneinduced leaf senescence depends on age-related changes and OLD genes in Arabidopsis. J Exp Bot 56:2915-2923

71. Koyama T (2014) The roles of ethylene and transcription factors in the regulation of onset of leaf senescence. Front Plant Sci 5:650

72. Wang KL, Li H, Ecker JR (2002) Ethylene biosynthesis and signaling networks. Plant Cell 14:131-152

73. Sharma P, Jha AB, Dubey RS, Pessarakli M (2012) Reactive oxygen species, oxidative damage, and antioxidative defense mechanism in plants under stressful conditions. J Bot 2012:217037

74. Mittler R (2017) ROS are good. Trends Plant Sci 22:11-19

75. Woo HR, Kim JH, Nam HF, Lim PO (2004) The delayed leaf senescence mutants of Arabidopsis, ore 1, ore 3, and ore 9 are tolerant to oxidative stress. Plant Cell Physiol 45:923-932

76. Schippers JHM, Nunes-Nesi A, Apetrei R, Hille J, Fernie AR, Dijkwel PP (2008) The Arabidopsis onset of leaf death5 mutation 
of quinolinate synthase affects nicotinamide adenine dinucleotide biosynthesis and causes early ageing. Plant Cell 20:2909-2925

77. Zentgraf U, Zimmermann P, Smykowski A (2012) Role of intracellular hydrogen peroxide as signalling molecule for plant senescence. In: Nagata T (ed) Senescence. InTech, London, pp $31-50$

78. Nishizawa-Yokoi A, Nosaka R, Hayashi H et al (2011) HsfA1d and HsfAle involved in the transcriptional regulation of Hsfa2 function as key regulators for the Hsf signaling network in response to environmental stress. Plant Cell Physiol 52:933-945

79. Wu A, Allu AD, Garapati $\mathrm{P}$ et al (2012) JUNGBRUNNEN1, a reactive oxygen species-responsive NAC transcription factor, regulates longevity in Arabidopsis. Plant Cell 24:482-506

80. Schramm F, Larkindale J, Kiehlmann E et al (2008) A cascade of transcription factor DREB2A and heat stress transcription factor HsfA3 regulates the heat stress response of Arabidopsis. Plant J 53:264-274

81. Yoshida T, Sakuma Y, Todaka D et al (2008) Functional analysis of an Arabidopsis heat-shock transcription factor $H s f A 3$ in the transcriptional cascade downstream of the DREB2A stress-regulatory system. Biochem Biophys Res Commun 368:515-521

82. Wang Z, Wang Y, Hong X et al (2015) Functional inactivation of UDP-N-acetylglucosamine pyrophosphorylase 1 (UAP1) induces early leaf senescence and defence responses in rice. J Exp Bot 66:973-987

83. Wu L, Ren D, Hu S et al (2016) Down-regulation of a nicotinate phosphoribosyltransferase gene, OsNaPRT1, leads to withered leaf tips. Plant Physiol 171:1085-1098

84. Hong Y, Zhang Y, Sinumporn S et al (2018) Premature leaf senescence 3, encoding a methyltransferase, is required for melatonin biosynthesis in rice. Plant J 95:877-891

85. Chen G, Wu C, He L et al (2018) Knocking out the gene RLSI induces hypersensitivity to oxidative stress and premature leaf senescence in rice. Int J Mol Sci 19:2853

86. Wang F, Liu J, Zhou L et al (2016) Senescence-specific change in ROS scavenging enzyme activities and regulation of various SOD isozymes to ROS levels in psf mutant rice leaves. Plant Physiol Biochem 109:248-261

87. Khorobrykh S, Havurinne V, Mattila H, Tyystjärvi E (2020) Oxygen and ROS in photosynthesis. Plants 9:91

88. Hörtensteiner S (2006) Chlorophyll degradation during senescence. Annu Rev Plant Biol 57:55-77

89. Schelbert S, Aubry S, Burla B et al (2009) Pheophytin pheophorbide hydrolase (pheophytinase) is involved in chlorophyll breakdown during leaf senescence in Arabidopsis. Plant Cell 21:767-785

90. Kusaba M, Ito H, Morita R et al (2007) Rice non-yellow coloring1 is involved in light-harvesting complex II and grana degradation during leaf senescence. Plant Cell 19:1362-1375

91. Sato Y, Morita R, Katsuma S, Nishimura M, Tanaka A, Kusaba M (2009) Two short-chain dehydrogenase/reductases, NONYELLOW COLORING 1 and NYC1-LIKE, are required for chlorophyll $\mathrm{b}$ and light-harvesting complex II degradation during senescence in rice. Plant J 57:120-131

92. Cha KW, Lee YJ, Koh HJ, Lee BM, Nam YW, Paek NC (2002) Isolation, characterization, and mapping of the stay green mutant in rice. Theor Appl Genet 104:526-532

93. Jiang $\mathrm{H}, \mathrm{Li} \mathrm{M}$, Liang $\mathrm{N}$ et al (2007) Molecular cloning and function analysis of the stay green gene in rice. Plant J 52:197-209

94. Park SY, Yu JW, Park JS et al (2007) The senescence-induced staygreen protein regulates chlorophyll degradation. Plant Cell 19:1649-1664

95. Zhou C, Han L, Pislariu C et al (2011) From model to crop: Functional analysis of a stay-green gene in the model legume
Medicago truncatula and effective use of the gene for alfalfa improvement. Plant Physiol 157:1483-1496

96. Ren G, An K, Liao Y et al (2007) Identification of a novel chloroplast protein AtNYE1 regulating chlorophyll degradation during leaf senescence in Arabidopsis. Plant Physiol 144:1429-1441

97. Sakuraba Y, Lee SH, Kim YS, Park OK, Hörtensteiner S, Paek NC (2014) Delayed degradation of chlorophylls and photosynthetic proteins in Arabidopsis autophagy mutants during stressinduced leaf yellowing. J Exp Bot 65:3915-3925

98. Li S, Gao J, Yao L et al (2016) The role of ANAC072 in the regulation of chlorophyll degradation during age- and dark-induced leaf senescence. Plant Cell Rep 35:1729-1741

99. Oda-Yamamizo C, Mitsuda N, Sakamoto S, Ogawa D, OhmeTakagi M, Ohmiya A (2016) The NAC transcription factor ANAC046 is a positive regulator of chlorophyll degradation and senescence in Arabidopsis leaves. Sci Rep 6:23609

100. Sakuraba Y, Han SH, Lee SH, Hörtensteiner S, Paek NC (2016) Arabidopsis NAC016 promotes chlorophyll breakdown by directly upregulating STAYGREEN1 transcription. Plant Cell Rep 35:155-166

101. Spano G, Di Fonzo N, Perrotta C et al (2003) Physiological characterization of "stay green" mutants in durum wheat. J Exp Bot 54:1415-1420

102. Ciereszko I (2018) Regulatory roles of sugars in plant growth and development. Acta Soc Bot Pol 87:3583

103. Lastdrager J, Hanson J, Smeekens S (2014) Sugar signals and the control of plant growth and development. J Exp Bot 65:799-807

104. Yu S, Lian H, Wang JW (2015) Plant developmental transitions: The role of microRNAs and sugars. Curr Opin Plant Biol 27:1-7

105. Yang L, Xu M, Koo Y, He J, Poethig SR (2013) Sugar promotes vegetative phase change in Arabidopsis thaliana by repressing the expression of MIR156A and MIR156C. Elife 2:e00260

106. Wang M, Zhang T, Peng $\mathrm{H}$ et al (2018) Rice premature leaf senescence 2 , encoding a glycosyltransferase (GT), is involved in leaf senescence. Front Plant Sci 26:560

107. Moore B, Zhou L, Rolland F et al (2003) Role of the Arabidopsis glucose sensor HXK1 in nutrient, light, and hormonal signaling. Science 300:332-336

108. Dai N, Schaffer A, Petreikov M et al (1999) Overexpression of Arabidopsis hexokinase in tomato plants inhibits growth, reduces photosynthesis, and induces rapid senescence. Plant Cell 11:1253-1266

109. Kim J (2019) Sugar metabolism as input signals and fuel for leaf senescence. Genes Genom 41:737-746

110. Baena-González E, Rolland F, Thevelein JM, Sheen J (2007) A central integrator of transcription networks in plant stress and energy signalling. Nature 448:938-942

111. Thimann KV, Tetley RM, Krivak BM (1977) Metabolism of oat leaves during senescence. Plant Physiol 54:294-303

112. Oh SA, Lee SY, Chung IK, Lee CH, Nam HG (1996) A senescence-associated gene of Arabidopsis thaliana is distinctively regulated during natural and artificially induced leaf senescence. Plant Mol Biol 30:739-754

113. Chung BC, Lee SY, Sung Aeong Oh, Rhew TH, Nam HG, Lee $\mathrm{CH}$ (1997) The promoter activity of sen 1, a senescence-associated gene of Arabidopsis, is repressed by sugars. J Plant Physiol 151:339-345

114. Jalil SU, Khan MIR, Ansari MI (2019) Role of GABA transaminase in the regulation of development and senescence in Arabidopsis thaliana. Curr Plant Biol 19:100119

115. Ahmed S, Ariyaratne M, Patel J et al (2017) Altered expression of polyamine transporters reveals a role for spermidine in the timing of flowering and other developmental response pathways. Plant Sci 258:146-155 
116. Alcázar R, García-Martínez JL, Cuevas JC, Tiburcio AF, Altabella T (2005) Overexpression of $A D C 2$ in Arabidopsis induces dwarfism and late-flowering through GA deficiency. Plant $\mathrm{J}$ 43:425-436

117. Sequera-Mutiozabal MI, Erban A, Kopka J et al (2016) Global metabolic profiling of Arabidopsis polyamine oxidase 4 (AtPAO4) loss-of-function mutants exhibiting delayed darkinduced senescence. Front Plant Sci 7:173

118. Sun L, Wang Y, Liu LL et al (2017) Isolation and characterization of a spotted leaf 32 mutant with early leaf senescence and enhanced defense response in rice. Sci Rep 7:41846

119. Riha K, McKnight TD, Griffing LR, Shippen DE (2001) Living with genome instability: plant responses to telomere dysfunction. Science 291:1797-1800

120. Kakutani T, Jeddeloh JA, Flowers SK, Munakata K, Richards EJ (1996) Developmental abnormalities and epimutations associated with DNA hypomethylation mutations. Proc Natl Acad Sci USA 93:12406-12411

121. Yao Y, Bilichak A, Golubov A, Kovalchuk I (2012) ddm1 plants are sensitive to methyl methane sulfonate and $\mathrm{NaCl}$ stresses and are deficient in DNA repair. Plant Cell Rep 31:1549-1561

122. Wang Y, Xiao R, Wang $\mathrm{H}$ et al (2014) The Arabidopsis RAD51 paralogs $R A D 51 B, R A D 51 D$ and $X R C C 2$ play partially redundant roles in somatic DNA repair and gene regulation. New Phytol 201:292-304

123. Bagherieh-Najjar MB, De Vries OMH, Hille J, Dijkwel PP (2005) Arabidopsis RecQl4A suppresses homologous recombination and modulates DNA damage responses. Plant J 43:789-798

124. Li Z, Kim JH, Kim J et al (2020) ATM suppresses leaf senescence triggered by DNA double-strand break through epigenetic control of senescence-associated genes in Arabidopsis. New Phytol 227:473-484

125. Santner A, Calderon-Villalobos LIA, Estelle M (2009) Plant hormones are versatile chemical regulators of plant growth. Nat Chem Biol 5:301-307

126. Davies PJ (2010) The plant hormones: their nature, occurrence, and functions. Plant hormones. Springer, Dordrecht, pp 1-15

127. Khan M, Rozhon W, Poppenberger B (2014) The role of hormones in the aging of plants-a mini-review. Gerontology 60:49-55

128. Eckardt NA (2015) The plant cell reviews dynamic aspects of plant hormone signaling and crosstalk. Plant Cell 27:1-2

129. Asami T, Nakagawa Y (2018) Preface to the special issue: brief review of plant hormones and their utilization in agriculture. $\mathrm{J}$ Pestic Sci 43:154-158

130. Wang YH, Irving HR (2011) Developing a model of plant hormone interactions. Plant Signal Behav 6:494-500

131. Sarwat M, Naqvi AR, Ahmad P, Ashraf M, Akram NA (2013) Phytohormones and microRNAs as sensors and regulators of leaf senescence: assigning macro roles to small molecules. Biotechnol Adv 31:1153-1171

132. Schippers JHM, Jing H, Hille J, Dijkwel PP (2007) Developmental and hormonal control of leaf senescence. Annu Plant Rev 26:145-170
133. Quirino BF, Normanly J, Amasino RM (1999) Diverse range of gene activity during Arabidopsis thaliana leaf senescence includes pathogen-independent induction of defense-related genes. Plant Mol Biol 40:267-278

134. Hansen H, Grossmann K (2000) Auxin-induced ethylene triggers abscisic acid biosynthesis and growth inhibition. Plant Physiol 124:1437-1448

135. Hou K, Wu W, Gan S-S (2013) SAUR36, a small auxin up RNA gene, is involved in the promotion of leaf senescence in Arabidopsis. Plant Physiol 161:1002-1009

136. Lim PO, Lee IC, Kim J et al (2010) Auxin response factor 2 (ARF2) plays a major role in regulating auxin-mediated leaf longevity. J Exp Bot 61:1419-1430

137. Ellis CM, Nagpal P, Young JC, Hagen G, Guilfoyle TJ, Reed JW (2005) Auxin response factor1 and auxin response factor2 regulate senescence and floral organ abscission in Arabidopsis thaliana. Development 132:4563-4574

138. Noh YS, Amasino RM (1999) Identification of a promoter region responsible for the senescence-specific expression of SAG12. Plant Mol Biol 41:181-194

139. Kim JI, Murphy AS, Baek D et al (2011) YUCCA6 over-expression demonstrates auxin function in delaying leaf senescence in Arabidopsis thaliana. J Exp Bot 62:3981-3192

140. Medford JI, Horgan R, El-Sawi Z, Klee HJ (1989) Alterations of endogenous cytokinins in transgenic plants using a chimeric isopentenyl transferase gene. Plant Cell 1(403):413

141. Smart C, Scofield S, Bevan M, Dyer T (1991) Delayed leaf senescence in tobacco plants transformed with $t m r$, a gene for cytokinin production in Agrobacterium. Plant Cell 3:647-656

142. Li Y, Hagen G, Guilfoyle TJ (1992) Altered morphology in transgenic tobacco plants that overproduce cytokinins in specific tissues and organs. Dev Biol 153:386-395

143. Wang J, Letham DS, Cornish E, Stevenson KR (1997) Studies of cytokinin action and metabolism using tobacco plants expressing either the ipt or the GUS gene controlled by a chalcone synthase promoter. I. Developmental features of the transgenic plants. Aust J Plant Physiol 24:661-672

144. Singh S, Letham DS, Palni LMS (1992) Cytokinin biochemistry in relation to leaf senescence. VIII. Translocation, metabolism and biosynthesis of cytokinins in relation to sequential leaf senescence of tobacco. Physiol Plant 86:398-406

145. Towne G, Owensby C (1983) Cytokinins effect on protein and chlorophyll content of big bluestem leaves. J Range Manag 36:1969-1971

146. Gan S, Amasino RM (1995) Inhibition of leaf senescence by autoregulated production of cytokinin. Science 270:1986-1988

Publisher's Note Springer Nature remains neutral with regard to jurisdictional claims in published maps and institutional affiliations. 